\title{
Periodic Rectangular and Circular Profiles in the Cross Section of the Straight Waveguide Based on Laplace and Fourier Transforms and Their Inverse Transforms and Applications
}

\author{
Zion Menachem \\ Additional information is available at the end of the chapter \\ http://dx.doi.org/10.5772/intechopen.76794
}

\begin{abstract}
This chapter presents propagation along the straight rectangular waveguide with periodic rectangular and circular profiles in the cross section. The objectives in this study are to explore the effect of the periodic rectangular and circular profiles in the cross section of the straight waveguide on the output field and to develop the technique to calculate two kinds of the periodic profiles. The method is based on Laplace and Fourier transforms and the inverse Laplace and Fourier transforms. The contribution of the proposed technique is important to improve the method that is based on Laplace and Fourier transforms and their inverse transforms also for the discontinuous periodic rectangular and circular profiles in the cross section (and not only for the continuous profiles). The proposed technique is very effective to solve complex problems, in relation to the conventional methods, especially when we have a large numbers of dielectric profiles. The application is useful for straight waveguides in the microwave and the millimeter wave regimes, with periodic rectangular and circular profiles in the cross section of the straight waveguide.
\end{abstract}

Keywords: wave propagation, helical waveguide, dielectric waveguide, power transmission 


\section{Introduction}

Review of numerical and approximate methods for the modal analysis of general optical dielectric waveguides with emphasis on recent developments has been published [1]. Examples of interesting methods, such as the finite difference method and the finite element method have been reviewed. The method for the eigenmode analysis of two-dimensional step-index waveguides has been proposed [2]. The method distinguishes itself other existing interface methods by avoiding the use of the Taylor series expansion and by introducing the concept of the iterative use of low-order jump conditions.

The method of selective suppression of electromagnetic modes in a rectangular waveguides by using distributed wall losses has been proposed [3]. Analytical design method for corrugated rectangular waveguide has been proposed [4].

A Fourier operator method has been used to derive for the first time an exact closed-form eigenvalue equation for the scalar mode propagation constants of a buried rectangular dielectric waveguide [5]. Wave propagation in an inhomogeneous transversely magnetized rectangular waveguide has been studied with the aid of a modified Sturm-Liouville differential equation [6]. A fundamental and accurate technique to compute the propagation constant of waves in a lossy rectangular waveguide has been proposed [7]. This method is based on matching the electric and magnetic fields at the boundary and allowing the wave numbers to take complex values.

A great amount of numerical results for cylindrical dielectric waveguide array have been presented [8]. Dielectric cylinders have been arrayed by a rectangular mode. When the area of dielectric cylinder in a unit cell varied from a small number to a big one and even maximum, interactions between space harmonics firstly got stronger but finally got weaker. Full-wave analysis of dielectric rectangular waveguides has been presented [9]. The waveguide properties of permeable one-dimensional periodic acoustic structures have been studied [10]. Analysis of rectangular folded-waveguide millimeter wave slow-wave structure using conformal transformation has been developed [11].

A simple closed-form expression to compute the time-domain reflection coefficient for a transient $T E_{10}$ mode wave incident on a dielectric step discontinuity in a rectangular waveguide has been presented [12]. In this paper, an exponential series approximation was provided for efficient computation of the reflected and transmitted field waveforms.

A waveguide with layered-periodic walls for different relations between the dielectric permittivities of the central layer and the superlattice layers has been proposed [13]. A full-vectorial boundary integral equation method for computing guided modes of optical waveguides has been presented [14]. A method for the propagation constants of arbitrary cross-sectional shapes has been described [15]. Experiment and simulation of $T E_{10}$ cut-off reflection phase in gentle rectangular downtapers has been studied [16].

The rectangular dielectric waveguide technique for the determination of complex permittivity of a wide class of dielectric materials of various thicknesses and cross sections has been described [17]. In this paper, the technique has been presented to determine the dielectric constant of materials. Fourier decomposition method applied to mapped infinite domains has 
been described [18], and this method is reliable down to modal cutoff. Analysis for new types of waveguide with Fourier's expansion differential method has been proposed [19].

Propagation characteristics of modes in some rectangular waveguides using the finitedifference time-domain method have been proposed [20]. Analysis of rectangular waveguide using finite element method has been presented for arbitrarily shaped waveguide [21]. Wave propagation and dielectric permittivity reconstruction in the case of a rectangular waveguide have been studied [22].

Important method for the analysis of electromagnetic wave propagation along the straight dielectric waveguide with arbitrary profiles has been proposed [23]. The mode model method for wave propagation in the straight waveguide with a circular cross section has been proposed [24]. This method in Refs. [23, 24] related to the methods based on Laplace and Fourier transforms and the inverse Laplace and Fourier transforms.

The objectives in this chapter are to explore the effect of the periodic rectangular and circular profiles in the cross section of the straight waveguide on the output field and to develop the technique to calculate the dielectric profile, the elements of the matrix, and its derivatives of the dielectric profile. The proposed technique is important to improve the mode model also for the periodic rectangular and circular profiles and not only for the continuous profiles.

\section{Periodic rectangular and circular profiles in the cross section of the straight waveguide}

In this chapter we introduce two different techniques, and the particular applications allow us to improve the mode model so that we can solve inhomogeneous problems also for periodic profiles in the cross section of the straight waveguide. Thus, in this chapter we introduce two techniques to calculate the dielectric profile, the elements of the matrix, and its derivatives of dielectric profile in the cases of periodic rectangular and circular profiles in the cross section of the straight rectangular waveguide.

The proposed techniques are very effective in relation to the conventional methods because they allow the development of expressions in the cross section only according to the specific discontinuous problem. In this way, the mode model method becomes an improved method to solve discontinuous problems in the cross section (and not only for continuous problems).

Three examples of periodic rectangular profiles are shown in Figure 1(a-c), and three examples of periodic circular profiles are shown in Figure 1(d-f) in the cross section of the straight rectangular waveguide.

An example of periodic structure with two rectangular profiles along x-axis is shown in Figure 1(a), where the centers of the left rectangle and right rectangle are located the points $(0.25 \mathrm{a}, 0.5 \mathrm{~b})$ and $(0.75 \mathrm{a}, 0.5 \mathrm{~b})$, respectively. An example of periodic structure with two rectangular profiles along y-axis is shown in Figure 1(b), where the centers of the upper rectangle and lower rectangle are located at the points $(0.5 \mathrm{a}, 0.75 \mathrm{~b})$ and $(0.5 \mathrm{a}, 0.25 \mathrm{~b})$.

An example of periodic structure with four rectangular profiles along $\mathrm{x}$-axis and $\mathrm{y}$-axis is shown in Figure 1(c). The center of the first rectangle is located at the point $(0.25 \mathrm{a}, 0.25 \mathrm{~b})$, 


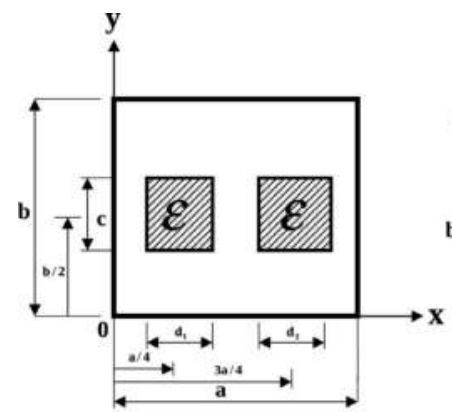

(a)

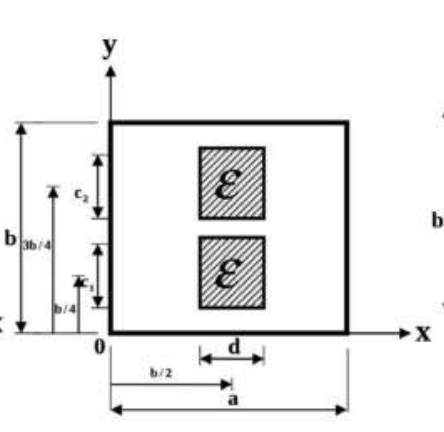

(b)

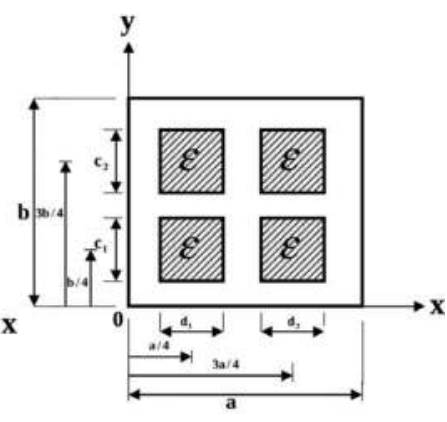

(c)

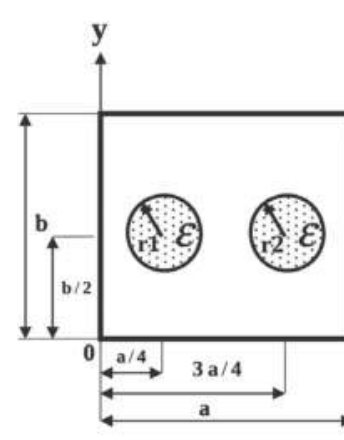

(d)

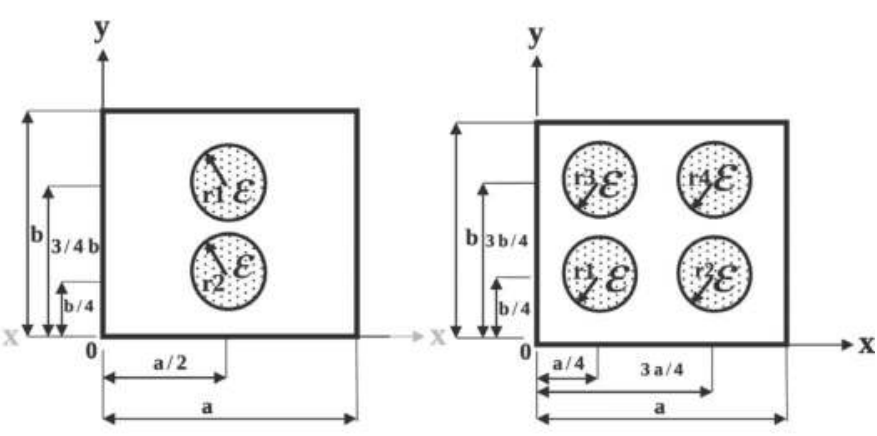

(e)

(f)

Figure 1. Examples of the periodic rectangular profiles $(\mathrm{a}-\mathrm{c})$ and periodic circular profiles $(\mathrm{d}-\mathrm{f})$ in the cross section of the straight rectangular waveguide.

the center of the second rectangle is located at the point $(0.75 \mathrm{a}, 0.25 \mathrm{~b})$, the center of the third rectangle is located at the point $(0.25 \mathrm{a}, 0.75 \mathrm{~b})$, and the center of the fourth rectangle is located at the point $(0.75 \mathrm{a}, 0.75 \mathrm{~b})$.

An example of periodic structure with two circular profiles along x-axis is shown in Figure 1(d), where the centers of the left circle and right circle are located at the points $(0.25 \mathrm{a}, 0.5 \mathrm{~b})$ and $(0.75$ a, $0.5 \mathrm{~b}$ ). An example of periodic structure with two circular profiles along y-axis is shown in Figure 1(e), where the centers of the upper circle and lower circle are located at the points $(0.5 \mathrm{a}$, $0.75 \mathrm{~b})$ and $(0.5 \mathrm{a}, 0.25 \mathrm{~b})$.

An example of periodic structure with four circular profiles along $\mathrm{x}$-axis and $\mathrm{y}$-axis is shown in Figure 1(f). The center of the first circle is located at the point $(0.25 \mathrm{a}, 0.25 \mathrm{~b})$, the center of the second circle is located at the point $(0.75 \mathrm{a}, 0.25 \mathrm{~b})$, the center of the third circle is located at the point $(0.25 \mathrm{a}, 0.75 \mathrm{~b})$, and the center of the fourth circle is located at the point $(0.75 \mathrm{a}, 0.75 \mathrm{~b})$.

The objective of this chapter is to introduce two different techniques that allow us to improve the model so that we can solve nonhomogeneous problems also for periodic profiles in the 
cross section of the straight rectangular waveguide. Thus, we need to introduce a technique and a particular application for the two geometric shapes composed of rectangles or circles in the cross section. We need to calculate the dielectric profile, the elements of the matrix, and its derivatives of the dielectric profile in the cases of periodic rectangular profiles (Figure 1(a-c)) and periodic circular profiles (Figure $\mathbf{1}(\mathbf{d}-\mathbf{f})$ ) in the cross section of the straight rectangular waveguide.

It is very interesting to compare between two different techniques of the two different kinds of the profiles (rectangular and circular) in the cross section of the rectangular straight waveguide.

\section{The techniques to solve two different profiles in the cross section}

The two kinds of the different techniques enable us to solve practical problems for the periodic rectangular profiles (Figure 1(a-c)) and periodic circular profiles (Figure $\mathbf{1}(\mathbf{d}-\mathbf{f})$ ) in the cross section of the straight rectangular waveguide.

Figure 2(a) shows one rectangular profile where the center of the rectangle is located at the point $(0.5 \mathrm{a}, 0.5 \mathrm{~b})$. Figure 2 (b) shows one circular profile where the center is located at the point $(0.5 \mathrm{a}, 0.5 \mathrm{~b})$. The proposed techniques to solve discontinuous problems with two different profiles (rectangular and circular) in the cross section will introduce according to Figure 2(a and $\mathbf{b})$.

Figure 2(a) shows one rectangular profile in the cross section, and Figure 2(b) shows one circular profile in the cross section. The dielectric profile $g(x, y)$ is given according to $\epsilon(x, y)=\epsilon_{0}(1+g(x, y))$. According to Figure 3(a and $\left.\mathbf{b}\right)$ and for $g(x, y)=g_{0}$, we obtain

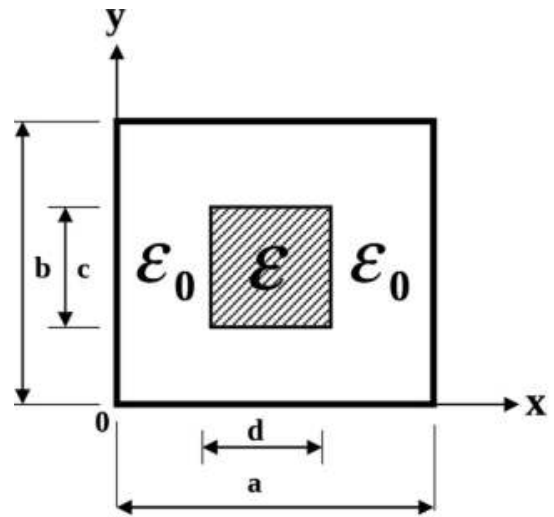

(a)

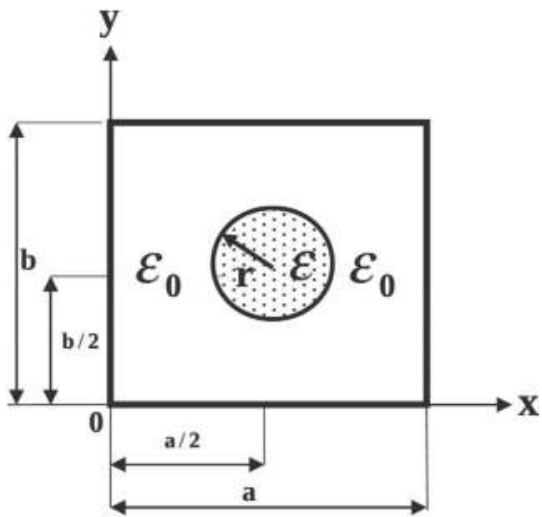

(b)

Figure 2. Examples of the rectangular and circular profiles in the cross section of the straight rectangular waveguide. (a) One rectangular profile. (b) One circular profile. 


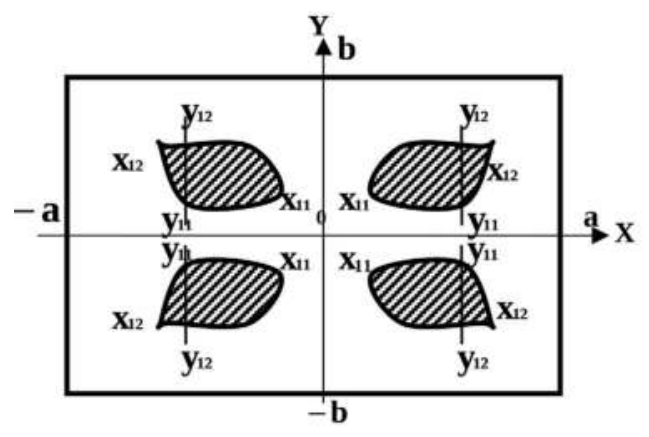

(a)

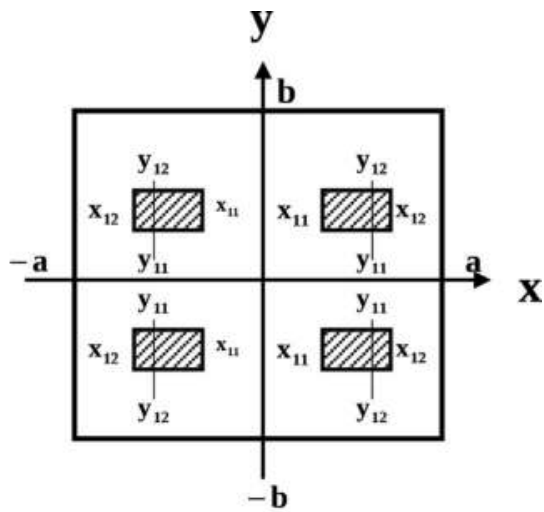

(b)

Figure 3. (a) The arbitrary profile in the cross section. (b) The rectangular profile in the cross section, as shown in Figure 2(a).

$$
\begin{aligned}
g(n, m)= & \frac{g_{0}}{4 a b}\left\{\int_{x_{11}}^{x_{12}} d x \int_{y_{11}}^{y_{12}} \exp \left(-j\left(k_{x} x+k_{y} y\right)\right) d y+\int_{-x_{12}}^{-x_{11}} d x \int_{y_{11}}^{y_{12}} \exp \left(-j\left(k_{x} x+k_{y} y\right)\right) d y\right. \\
& \left.+\int_{-x_{12}}^{-x_{11}} d x \int_{-y_{12}}^{-y_{11}} \exp \left(-j\left(k_{x} x+k_{y} y\right)\right) d y+\int_{x_{11}}^{x_{12}} d x \int_{-y_{12}}^{-y_{11}} \exp \left(-j\left(k_{x} x+k_{y} y\right)\right) d y\right\} .
\end{aligned}
$$

If $y_{11}$ and $y_{12}$ are not functions of $x$, then the dielectric profile is given by

$$
g(n, m)=\frac{g_{0}}{a b} \int_{x_{11}}^{x_{12}} \cos \left(k_{x} x\right) d x \int_{y_{11}}^{y_{12}} \cos \left(k_{y} y\right) d y
$$

The derivative of the dielectric profile in the case of $y_{11}$ and $y_{12}$ which are functions of $x$ is given by

$$
g_{x}(n, m)=\frac{2}{a m \pi} \int_{x_{11}}^{x_{12}} g_{x}(x, y) \sin \left[\frac{k_{y}}{2}\left(y_{12}-y_{11}\right)\right] \cos \left[\frac{k_{y}}{2}\left(y_{12}+y_{11}\right)\right] \cos \left(k_{x} x\right) d x
$$

where $g_{x}(x, y)=(1 / \epsilon(x, y))(d \epsilon(x, y) / d x), \epsilon(x, y)=\epsilon_{0}(1+g(x, y)), k_{x}=(n \pi x) / a$, and $k_{y}=(m \pi y) / b$. Similarly, we can calculate the value of $g_{y}(n, m)$, where $g_{y}(x, y)=(1 / \epsilon(x, y))(d \epsilon(x, y) / d y)$.

For the cross section as shown in Figure 2(a) and according to Figure 3(b), the center of the rectangle is located at $(0.5 a, 0.5 b), y_{12}=b / 2+c / 2$, and $y_{11}=b / 2-c / 2$. Thus, for this case, $y_{12}-y_{11}=\mathrm{c}$ and $y_{12}+y_{11}=\mathrm{b}$. In the same principle, the location of the rectangle should be taken into account. 
The particular application is based on the $\omega_{\varepsilon}$ function [25]. The $\omega_{\varepsilon}$ function is used in order to solve rectangular profile, periodic rectangular profiles, circular profile, and periodic circular profile in the cross section of the straight waveguide. The $\omega_{\varepsilon}$ function is defined as $\omega_{\varepsilon}(r)=C_{\varepsilon} \exp \left[-\varepsilon^{2} /\left(\varepsilon^{2}-|r|^{2}\right)\right]$ for $|r|>\varepsilon$, where $C_{\varepsilon}$ is a constant and $\int \omega_{\varepsilon}(r) d r=1$. In the limit $\varepsilon \rightarrow 0$, the $\omega_{\varepsilon}$ function is shown in Figure 4 .

The technique based on $\omega_{\varepsilon}$ function is very effective to solve complex problems, in relation to the conventional methods, especially when we have a large number of dielectric profiles, as shown in Figure 1(c and $\mathbf{f})$. We will demonstrate how to use with the proposed technique for all the cases that are shown in the examples of Figure 1(a-f).

\subsection{The technique based on $\omega_{\varepsilon}$ function for the periodic rectangular profile in the cross section}

Figure 1(a) shows the periodic rectangular profile where the center of the left rectangle is located at $(0.25 \mathrm{a}, 0.5 \mathrm{~b})$ and the right rectangle is located at $(0.75 \mathrm{a}, 0.5 \mathrm{~b})$. This dielectric profile is given by

$$
g(x)= \begin{cases}g_{0} \exp \left(1-q_{1}(x)\right) & \left((a / 2)-d_{1}-\varepsilon\right) / 2 \leq x<\left((a / 2)-d_{1}+\varepsilon\right) / 2 \\ g_{0} & \left((a / 2)-d_{1}+\varepsilon\right) / 2<x<\left((a / 2)+d_{1}-\varepsilon\right) / 2 \\ g_{0} \exp \left(1-q_{2}(x)\right) & \left((a / 2)+d_{1}-\varepsilon\right) / 2 \leq x<\left((a / 2)+d_{1}+\varepsilon\right) / 2 \\ g_{0} \exp \left(1-q_{3}(x)\right) & \left((3 a / 2)-d_{2}-\varepsilon\right) / 2 \leq x<\left((3 a / 2)-d_{2}+\varepsilon\right) / 2 \\ g_{0} & \left((3 a / 2)-d_{2}+\varepsilon\right) / 2<x<\left((3 a / 2)+d_{2}-\varepsilon\right) / 2 \\ g_{0} \exp \left(1-q_{4}(x)\right) & \left((3 a / 2)+d_{2}-\varepsilon\right) / 2 \leq x<\left((3 a / 2)+d_{2}+\varepsilon\right) / 2 \\ 0 & \text { else }\end{cases}
$$

and

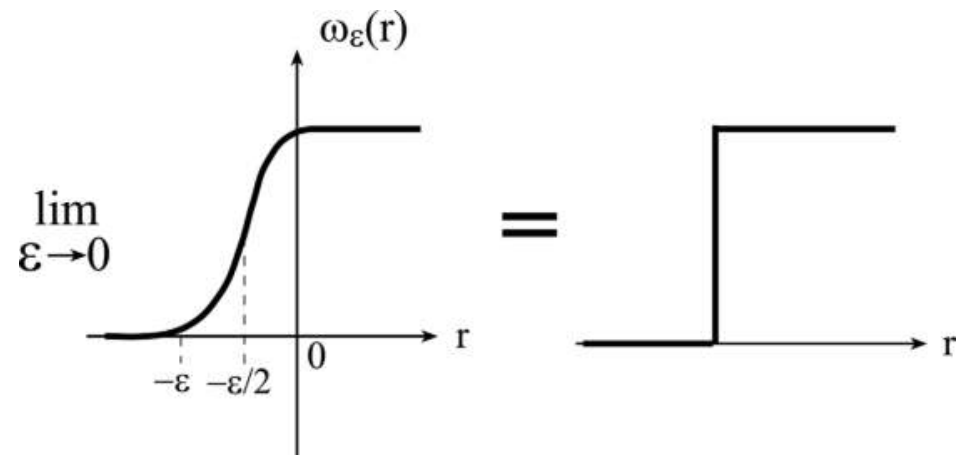

Figure 4. The technique based on $\omega_{\varepsilon}$ function in the limit $\varepsilon \rightarrow 0$ to solve discontinuous problems. 


$$
g(y)= \begin{cases}g_{0} \exp \left(1-q_{5}(y)\right) & (b-c-\varepsilon) / 2 \leq y<(b-c+\varepsilon) / 2 \\ g_{0} & (b-c+\varepsilon) / 2<y<(b+c-\varepsilon) / 2 \\ g_{0} \exp \left(1-q_{6}(y)\right) & (b+c-\varepsilon) / 2 \leq y<(b+c+\varepsilon) / 2 \\ 0 & \text { else }\end{cases}
$$

where

$$
\begin{array}{ll}
q_{1}(x)=\frac{\varepsilon^{2}}{\varepsilon^{2}-\left[x-\left((a / 2)-d_{1}+\varepsilon\right) / 2\right]^{2}} & , \quad q_{2}(x)=\frac{\varepsilon^{2}}{\varepsilon^{2}-\left[x-\left((a / 2)+d_{1}-\varepsilon\right) / 2\right]^{2}} \\
q_{3}(x)=\frac{\varepsilon^{2}}{\varepsilon^{2}-\left[x-\left((3 a / 2)-d_{2}+\varepsilon\right) / 2\right]^{2}} & , \quad q_{4}(x)=\frac{\varepsilon^{2}}{\varepsilon^{2}-\left[x-\left((3 a / 2)+d_{2}-\varepsilon\right) / 2\right]^{2}} \\
q_{5}(y)=\frac{\varepsilon^{2}}{\varepsilon^{2}-[y-(b-c+\varepsilon) / 2]^{2}} \quad, \quad & q_{6}(y)=\frac{\varepsilon^{2}}{\varepsilon^{2}-[y-(b+c-\varepsilon) / 2]^{2}} .
\end{array}
$$

The elements of the matrix are given in this case by

$$
\begin{aligned}
g(n, m)= & \frac{g_{0}}{a b}\left\{\int_{\left((a / 2)-d_{1}-\varepsilon\right) / 2}^{\left((a / 2)-d_{1}+\varepsilon\right) / 2} \exp \left(1-q_{1}(x)\right) \cos \left(\frac{n \pi x}{a}\right) d x+\right. \\
& \int_{\left((a / 2)-d_{1}+\varepsilon\right) / 2}^{\left((a / 2)+d_{1}-\varepsilon\right) / 2} \cos \left(\frac{n \pi x}{a}\right) d x+\int_{\left((a / 2)+d_{1}-\varepsilon\right) / 2}^{\left((a / 2)+d_{1}+\varepsilon\right) / 2} \exp \left(1-q_{2}(x)\right) \cos \left(\frac{n \pi x}{a}\right) d x+ \\
& \int_{\left((3 a / 2)-d_{2}-\varepsilon\right) / 2}^{\left((3 a / 2)-d_{2}+\varepsilon\right) / 2} \exp \left(1-q_{3}(x)\right) \cos \left(\frac{n \pi x}{a}\right) d x+ \\
& \left.\int_{\left((3 a / 2)-d_{2}+\varepsilon\right) / 2}^{\left((3 a / 2)+d_{2}-\varepsilon\right) / 2} \cos \left(\frac{n \pi x}{a}\right) d x+\int_{\left((3 a / 2)+d_{2}-\varepsilon\right) / 2}^{\left((3 a / 2)+d_{2}+\varepsilon\right) / 2} \exp \left(1-q_{4}(x)\right) \cos \left(\frac{n \pi x}{a}\right) d x\right\} \\
& \left\{\int_{(b-c-\varepsilon) / 2}^{(b-c+\varepsilon) / 2} \exp \left(1-q_{5}(y)\right) \cos \left(\frac{m \pi y}{b}\right) d y+\int_{(b-c+\varepsilon) / 2}^{(b+c-\varepsilon) / 2} \cos \left(\frac{m \pi y}{b}\right) d y+\right. \\
& \left.\int_{(b+c-\varepsilon) / 2}^{(b+c+\varepsilon) / 2} \exp \left(1-q_{6}(y)\right) \cos \left(\frac{m \pi y}{b}\right) d y\right\} .
\end{aligned}
$$

The derivatives of the dielectric profile are given in this case by

$$
g_{x}=\left\{\begin{array}{lll}
\frac{d}{d x}\left(\operatorname { l n } \left(1+g_{0}\right.\right. & \left.\left.q_{1}(x)\right)\right) & \left((a / 2)-d_{1}-\varepsilon\right) / 2 \leq x<\left((a / 2)-d_{1}+\varepsilon\right) / 2 \\
\frac{d}{d x}\left(\operatorname { l n } \left(1+g_{0}\right.\right. & \left.\left.q_{2}(x)\right)\right) & \left((a / 2)+d_{1}-\varepsilon\right) / 2 \leq x<\left((a / 2)+d_{1}+\varepsilon\right) / 2 \\
\frac{d}{d x}\left(\operatorname { l n } \left(1+g_{0}\right.\right. & \left.\left.q_{3}(x)\right)\right) & \left((3 a / 2)-d_{2}-\varepsilon\right) / 2 \leq x<\left((3 a / 2)-d_{2}+\varepsilon\right) / 2^{\prime} \\
\frac{d}{d x}\left(\operatorname { l n } \left(1+g_{0}\right.\right. & \left.\left.g_{4}(x)\right)\right) & \left((3 a / 2)+d_{2}-\varepsilon\right) / 2 \leq x<\left((3 a / 2)+d_{2}+\varepsilon\right) / 2 \\
0 & \text { else }
\end{array}\right.
$$


and

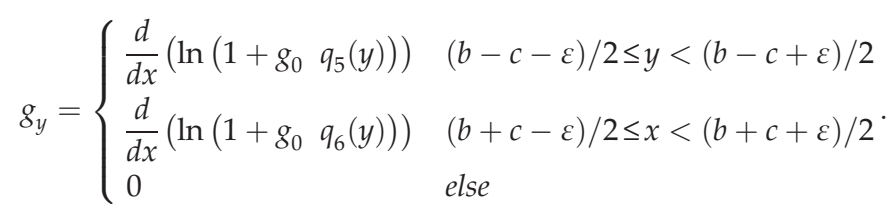

The derivative of the dielectric profile for $\mathrm{m}=0$ in the case of the periodic profile (Figure 1(a)) in the cross section of the waveguide is given in the limit $\varepsilon \rightarrow 0$ by

$$
\begin{aligned}
g_{x}(n, m=0)= & \frac{1}{a}\left\{\int_{\left((a / 2)-d_{1}-\varepsilon\right) / 2}^{\left((a / 2)-d_{1}+\varepsilon\right) / 2} \cos \left(k_{x} x\right) \frac{d}{d x}\left[\ln \left(1+g_{0} q_{1}(x)\right)\right] d x\right. \\
& +\int_{\left((a / 2)+d_{1}-\varepsilon\right) / 2}^{\left((a / 2)+d_{1}+\varepsilon\right) / 2} \cos \left(k_{x} x\right) \frac{d}{d x}\left[\ln \left(1+g_{0} q_{2}(x)\right)\right] d x \\
& +\int_{\left((3 a / 2)-d_{2}-\varepsilon\right) / 2}^{\left((3 a / 2)-d_{2}+\varepsilon\right) / 2} \cos \left(k_{x} x\right) \frac{d}{d x}\left[\ln \left(1+g_{0} q_{3}(x)\right)\right] d x \\
& \left.+\int_{\left((3 a / 2)+d_{2}-\varepsilon\right) / 2}^{\left((3 a / 2)+d_{2}+\varepsilon\right) / 2} \cos \left(k_{x} x\right) \frac{d}{d x}\left[\ln \left(1+g_{0} q_{4}(x)\right)\right] d x\right\} .
\end{aligned}
$$

Similarly, we can calculate the derivative of the dielectric profile for any value of $\mathrm{n}$ and $\mathrm{m}$.

\subsection{The technique based on $\omega_{\varepsilon}$ function for one circular profile in the cross section}

The dielectric profile for one circle is given where the center is located at $(0.5 \mathrm{a}, 0.5 \mathrm{~b})$ (Figure 2(b)) by

$$
g(x, y)= \begin{cases}g_{0} & 0 \leq r<r_{1}-\varepsilon_{1} / 2 \\ g_{0} \exp \left[1-q_{\varepsilon}(r)\right] & r_{1}-\varepsilon_{1} / 2 \leq r<r_{1}+\varepsilon_{1} / 2^{\prime}\end{cases}
$$

where

$$
q_{\epsilon}(r)=\frac{\varepsilon_{1}^{2}}{\varepsilon_{1}^{2}-\left[r-\left(r_{1}-\varepsilon_{1} / 2\right)\right]^{2}}
$$

else $g(x, y)=0$.

The radius of the circle is given by

$$
r=\sqrt{(x-a / 2)^{2}+(y-b / 2)^{2}} .
$$

Similarly, we can calculate the periodic circular profiles according to their location. 
Thus, the derivatives of the dielectric profile for one circle are given where the center is located at $(0.5 \mathrm{a}, 0.5 \mathrm{~b})$ (Figure 2(b)) in the region $r_{1}-\varepsilon_{1} / 2 \leq r<r_{1}+\varepsilon_{1} / 2$ by

$$
\begin{aligned}
& g_{x}=\frac{-2 g_{0} \cos \theta \exp \left[1-q_{\epsilon}(r)\right]\left[r-\left(r_{1}-\varepsilon_{1} / 2\right)\right] \varepsilon_{1}^{2}}{\left\{1+g_{0} \exp \left[1-q_{\epsilon}(r)\right]\right\}\left[\varepsilon_{1}{ }^{2}-\left[r-\left(r_{1}-\varepsilon_{1} / 2\right)\right]^{2}\right]^{2}}, \\
& g_{y}=\frac{-2 g_{0} \sin \theta \exp \left[1-q_{\epsilon}(r)\right]\left[r-\left(r_{1}-\varepsilon_{1} / 2\right)\right] \varepsilon_{1}{ }^{2}}{\left\{1+g_{0} \exp \left[1-q_{\epsilon}(r)\right]\right\}\left[\varepsilon_{1}{ }^{2}-\left[r-\left(r_{1}-\varepsilon_{1} / 2\right)\right]^{2}\right]^{2}},
\end{aligned}
$$

else $g_{x}=0$, and $g_{y}=0$.

The elements of the matrices for one circle are given where the center is located at $(0.5 \mathrm{a}, 0.5 \mathrm{~b})$ (Figure 2(b)) by

$$
\begin{gathered}
g(n, m)=\frac{g_{0}}{a b}\left\{\int_{0}^{2 \pi} \int_{0}^{r_{1}-\epsilon_{1} / 2} \cos \left[\frac{n \pi}{a}\left(r \cos \theta+\frac{a}{2}\right)\right] \cos \left[\frac{m \pi}{b}\left(r \sin \theta+\frac{b}{2}\right)\right]+\right. \\
\left.\int_{0}^{2 \pi} \int_{r_{1}-\epsilon_{1} / 2}^{r_{1}+\epsilon_{1} / 2} \cos \left[\frac{n \pi}{a}\left(r \cos \theta+\frac{a}{2}\right)\right] \cos \left[\frac{m \pi}{b}\left(r \sin \theta+\frac{b}{2}\right)\right] \exp \left[1-q_{\varepsilon}(r)\right]\right\} r d r d \theta, \\
g_{x}(n, m)=-\frac{2 g_{0}}{a b}\left\{\int_{0}^{2 \pi} \int_{r_{1}-\epsilon_{1} / 2}^{r_{1}+\epsilon_{1} / 2} \frac{\epsilon_{1}^{2}\left[r-\left(r_{1}-\epsilon_{1} / 2\right)\right] \exp \left[1-q_{\epsilon}(r)\right] \cos \theta}{\left[\epsilon_{1}^{2}-\left[r-\left(r_{1}-\epsilon_{1} / 2\right)\right]^{2}\right]^{2}\left[1+g_{0} \exp \left[1-q_{\epsilon}(r)\right]\right]}\right. \\
g_{y}(n, m)=-\frac{2 g_{0}}{a b}\left\{\int_{0}^{2 \pi} \int_{r_{1}-\epsilon_{1} / 2}^{r_{1}+\epsilon_{1} / 2} \frac{\epsilon_{1}^{2}\left[r-\left(r_{1}-\epsilon_{1} / 2\right)\right] \exp \left[1-q_{\epsilon}(r)\right] \sin \theta}{\left.\left[r-\left(r_{1}-\epsilon_{1} / 2\right)\right]^{2}\right]}\left[1+g_{0} \exp \left[1-q_{\epsilon}(r)\right]\right]\right. \\
\left.\cos \left[\frac{n \pi}{a}\left(r \cos \theta+\frac{a}{2}\right)\right] \cos \left[\frac{m \pi}{b}\left(r \sin \theta+\frac{b}{2}\right)\right]\right\} r d r d \theta
\end{gathered}
$$

where $r=\sqrt{(x-a / 2)^{2}+(y-b / 2)^{2}}$.

Similarly, we can calculate the periodic circular profile according to the number of the circles and the locations of their centers.

The matrix $\mathrm{G}$ is given by the form

$$
\mathbf{G}=\left[\begin{array}{lllllll}
g_{00} & g_{-10} & g_{-20} & \cdots & g_{-n m} & \cdots & g_{-N M} \\
g_{10} & g_{00} & g_{-10} & \ldots & g_{-(n-1) m} & \cdots & g_{-(N-1) M} \\
g_{20} & g_{10} & \ddots & \ddots & \ddots & & \\
\vdots & g_{20} & \ddots & \ddots & \ddots & & \\
g_{n m} & \ddots & \ddots & \ddots & g_{00} & \vdots & \\
\vdots & & & & & & \\
g_{N M} & \cdots & \cdots & \cdots & \cdots & \cdots & g_{00}
\end{array}\right] .
$$


Similarly, the $\mathrm{G}_{\mathbf{x}}$ and $\mathrm{G}_{\mathbf{y}}$ matrices are obtained by the derivatives of the dielectric profile. These matrices relate to the method that is based on the Laplace and Fourier transforms and their inverse transforms [23].

The technique is important to solve discontinuous periodic rectangular and circular profiles in the cross section of the straight waveguide. The graphical results in the next section will be demonstrated as a response to a half-sine $\left(T E_{10}\right)$ input-wave profile.

\section{Numerical results of periodic rectangular and circular dielectric materials}

All the graphical results are demonstrated as a response to a half-sine $\left(T E_{10}\right)$ input-wave profile for the periodic rectangular profiles (Figure 1(a-c)) and the periodic circular profiles (Figure 1(d-f)) in the cross section of the straight rectangular waveguide.

The output fields for the periodic structure with the two rectangular profiles along $x$-axis (Figure 1(a)) are demonstrated in Figure 5(a and b) for $\epsilon_{r}=3$ and for $\epsilon_{r}=10$, respectively. In this examples, the left rectangle is located at the point $(0.25 \mathrm{a}, 0.5 \mathrm{~b})$, and the center of the right rectangle is located at the point $(0.75 \mathrm{a}, 0.5 \mathrm{~b})$. Figure 5 (c) shows the output field as the function of x-axis where $\mathrm{y}=\mathrm{b} / 2=10 \mathrm{~mm}$ for four values of $\epsilon_{r}=3,5,7$, and 10 , respectively.

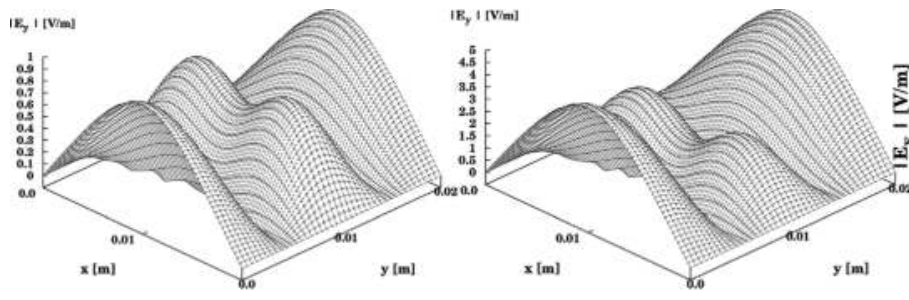

(a)

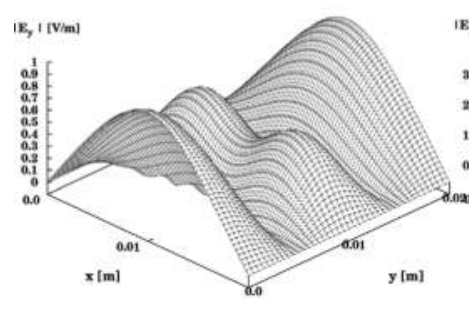

(d) (b)

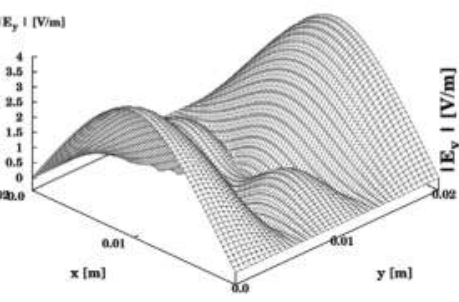

(e)

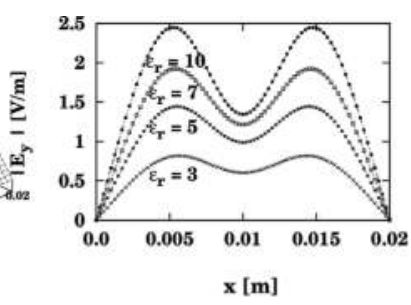

(c)

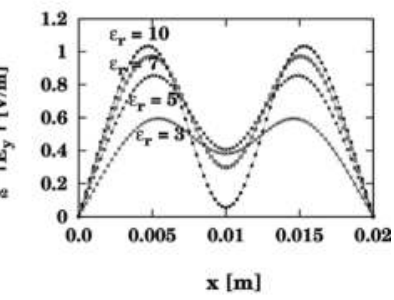

(f)

Figure 5. The output field as a response to a half-sine $\left(T E_{10}\right)$ input-wave profile for the periodic rectangular profiles (a-c) that relate to cross section (Figure 1(a)) and for the periodic circular profiles (d-f) that relate to cross section (Figure 1(d)). The results are shown for (a) $\epsilon_{r}=3$; (b) $\epsilon_{r}=10$; (c) $\epsilon_{r}=3,5,7$, and 10 ; (d) $\epsilon_{r}=3$; (e) $\epsilon_{r}=10$; and (f) $\epsilon_{r}=3,5,7$, and 10 . 
The parameters of these examples are $\mathrm{a}=2 \mathrm{~cm}, \mathrm{~b}=2 \mathrm{~cm}, \lambda=3.75 \mathrm{~cm}, \quad \beta=581 / \mathrm{m}$, $k_{0}=1671 / \mathrm{m}$, and $\mathrm{z}=15 \mathrm{~cm}$.

The output fields for the periodic structure with the two circular profiles along x-axis (Figure 1(d)) are demonstrated in Figure 5(d and e) for $\boldsymbol{\epsilon}_{r}=3$ and for $\boldsymbol{\epsilon}_{r}=10$, respectively. In this examples, the left circle is located at the point $(0.25 \mathrm{a}, 0.5 \mathrm{~b})$, and the center of the right circle is located at the point $(0.75 \mathrm{a}, 0.5 \mathrm{~b})$. Figure $5(\mathbf{f})$ shows the output field as the function of $\mathrm{x}$-axis where $\mathrm{y}=\mathrm{b} / 2=10 \mathrm{~mm}$ for four values of $\epsilon_{r}=3,5,7$, and 10, respectively.

It is interesting to see a similar behavior of the output results in the cases of periodic rectangular profiles (Figure 5(a-c)) that relate to Figure 1(a) and in the cases of periodic circular profiles (Figure $\mathbf{5}(\mathbf{d}-\mathbf{f})$ ) that relate to Figure $\mathbf{1}(\mathbf{d})$, where $\epsilon_{r}=3$ and 10, respectively. The behavior is similar, but not for every $\epsilon_{r}$, and the amplitudes of the output fields are different.

The output fields (Figure $5(a-f)$ ) are strongly affected by the input-wave profile ( $T E_{10}$ mode), the periodic structure with the two rectangular profiles or the two circular profiles along $x$-axis, and the distance between the two centers of the rectangular or the circular profiles.

The output fields for the periodic structure with the two rectangular profiles along $y$-axis (Figure 1(b)) are demonstrated in Figure 6(a-c) for $\epsilon_{r}=3,5$, and 10, respectively. The centers of the upper rectangle and the lower rectangle are located at the points $(0.5 \mathrm{a}, 0.75 \mathrm{~b})$ and

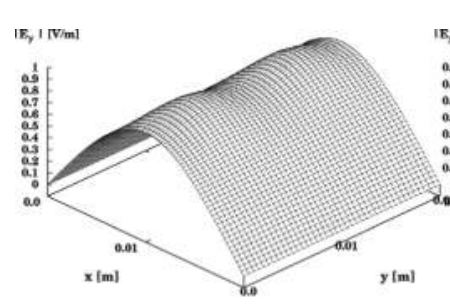

(a)

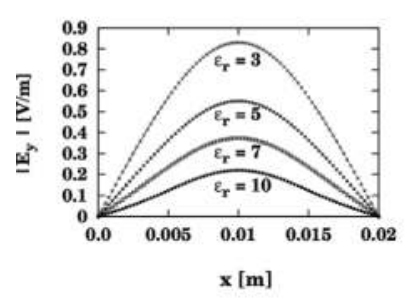

(d)

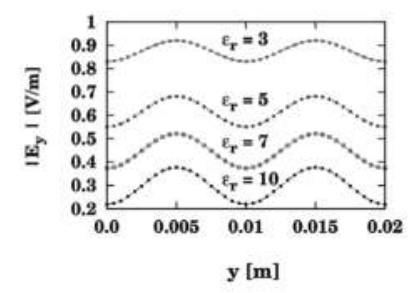

(e)

Figure 6. The output field as a response to a half-sine $\left(T E_{10}\right)$ input-wave profile for the periodic rectangular profiles (a-c) that relate to cross section (Figure 1(b)). The results are shown for (a) $\epsilon_{r}=3$; (b) $\epsilon_{r}=5$; (c) $\epsilon_{r}=10$; (d) $\epsilon_{r}=3,5,7$, and 10; and $(\mathrm{e}) \epsilon_{r}=3,5,7$, and 10 . 
$(0.5 \mathrm{a}, 0.25 \mathrm{~b})$, respectively. The output field is shown in Figure 6(d) for $x$-axis where $y=b / 2=$ $10 \mathrm{~mm}$ and is shown in Figure 6(e) for $\mathrm{y}$-axis where $\mathrm{x}=\mathrm{a} / 2=10 \mathrm{~mm}$ and for $\epsilon_{r}=3,5,7$, and 10, respectively.

The parameters of these examples are $\mathrm{a}=2 \mathrm{~cm}, \mathrm{~b}=2 \mathrm{~cm}, \mathrm{c}=2.5 \mathrm{~mm}, \mathrm{~d}=2.5 \mathrm{~mm}, \lambda=3.75 \mathrm{~cm}$, $\beta=581 / m, k_{0}=1671 / \mathrm{m}$, and $\mathrm{z}=15 \mathrm{~cm}$.

By changing only the parameter $\epsilon_{r}$ from 3 to 10, the relative profile of the output field is changed from a half-sine ( $\left.T E_{10}\right)$ profile to a Gaussian shape profile, as shown in Figure 6(d). The output field in Figure 6(e) demonstrates the periodic structure with the two rectangular profiles for $\epsilon_{r}=3,5,7$, and 10 .

The output fields for the periodic structure with the two circular profiles along y-axis (Figure 1(e)) are demonstrated in Figure $\mathbf{7}(\mathbf{a}-\mathbf{c})$ for $\epsilon_{r}=3,5$, and 10, respectively. The centers of the upper circle and the lower circle are located at the points $(0.5 \mathrm{a}, 0.75 \mathrm{~b})$ and $(0.5 \mathrm{a}, 0.25 \mathrm{~b})$, respectively. The output field is shown in Figure $7(\mathbf{d})$ for $x$-axis where $y=b / 2=10 \mathrm{~mm}$ and is shown in Figure 7(e) for $\mathrm{y}$-axis where $\mathrm{x}=\mathrm{a} / 2=10 \mathrm{~mm}$ for $\epsilon_{r}=3,5,7$, and 10, respectively.

By changing only the parameter $\epsilon_{r}$ from 3 to 10, the relative profile of the output field is changed from a half-sine $\left(T E_{10}\right)$ profile to a Gaussian shape profile. The output field in Figure 7 (e) demonstrates the periodic structure with the two circular profiles for $\epsilon_{r}=3,5,7$, and 10 .

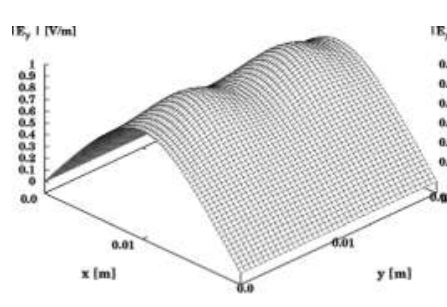

(a)

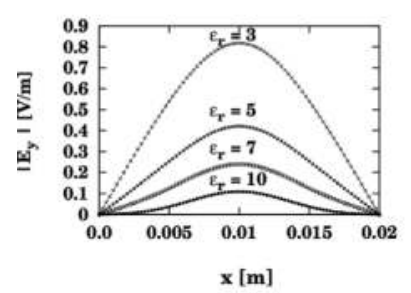

(d)

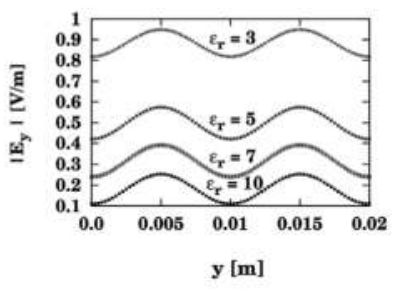

(e)

Figure 7. The output field as a response to a half-sine $\left(T E_{10}\right)$ input-wave profile for the periodic circular profiles $(\mathrm{a}-\mathrm{c})$ that relate to cross section (Figure 1(e)). The results are shown for (a) $\epsilon_{r}=3$; (b) $\epsilon_{r}=5$; (c) $\epsilon_{r}=10$; (d) $\epsilon_{r}=3,5,7$, and 10; and (e) $\epsilon_{r}=3,5,7$, and 10 . 
The parameters of these examples are $\mathrm{a}=2 \mathrm{~cm}, \mathrm{~b}=2 \mathrm{~cm}, \mathrm{c}=2.5 \mathrm{~mm}, \mathrm{~d}=2.5 \mathrm{~mm}, \lambda=3.75 \mathrm{~cm}$, $\beta=581 / \mathrm{m}, k_{0}=1671 / \mathrm{m}$, and $\mathrm{z}=15 \mathrm{~cm}$. The radius of the circle is equal to $2.5 \mathrm{~mm}$.

The output fields (Figure 6(a-e)) and Figure 7(a-e)) are strongly affected by the input-wave profile ( $T E_{10}$ mode), the periodic structure with the two rectangular profiles (Figure 1(b)) or circular profiles (Figure 1(e)) along y-axis, and the distance between the two centers of the profiles.

It is interesting to see a similar behavior of the output results in the cases of periodic rectangular profiles (Figure 6(a-e)) that relate to Figure 1(b) and in the cases of periodic circular profiles (Figure 7(a-e)) that relate to Figure 1(e), for every value of $\epsilon_{r}$, respectively. According to these output results, we see the similar behavior for every value of $\epsilon_{r}$, but the amplitudes of the output fields are different.

The output fields for the periodic structure with four rectangular profiles along $\mathrm{x}$-axis and $\mathrm{y}$-axis (Figure 1(c)) are demonstrated in Figure 8(a and $\mathbf{b})$ for $\epsilon_{r}=1.2$ and 1.4, respectively. The center of the first rectangle is located at the point $(0.25 \mathrm{a}, 0.25 \mathrm{~b})$, the center of the second rectangle is located at the point $(0.75 \mathrm{a}, 0.25 \mathrm{~b})$, the center of the third rectangle is located at the point $(0.25 \mathrm{a}, 0.75 \mathrm{~b})$, and the center of the firth rectangle is located at the point $(0.75 \mathrm{a}, 0.75 \mathrm{~b})$.

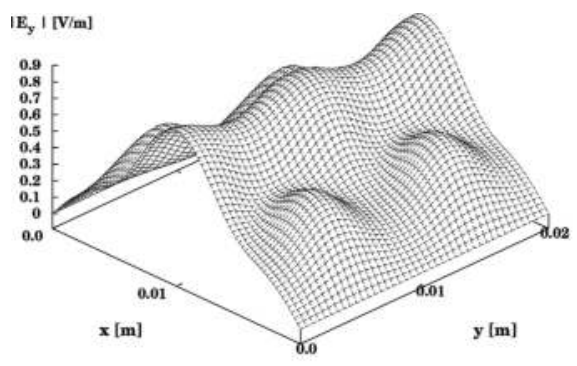

(a)

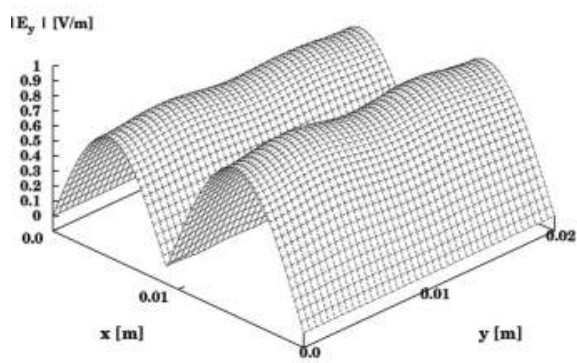

(c)

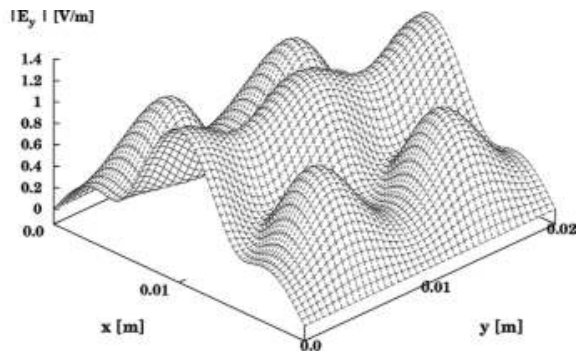

(b)

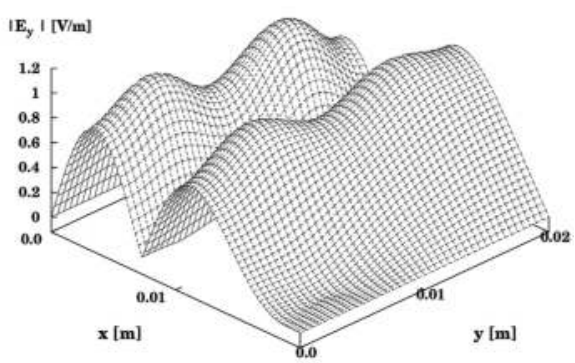

(d)

Figure 8. The output field as a response to a half-sine $\left(T E_{10}\right)$ input-wave profile in the case of four rectangular profiles along $\mathrm{x}$-axis and along y-axis (Figure 1(c)) for (a) $\epsilon_{r}=1.2$ and (b) $\epsilon_{r}=1.4$. The output field as a response to a half-sine $\left(T E_{10}\right)$ input-wave profile in the case of four circular profiles along x-axis and along y-axis (Figure 1(f)) for (c) $\epsilon_{r}=4$ and (b). $\epsilon_{r}=10$. 
By increasing the parameter $\epsilon_{r}$ from 1.2 to 1.4, the output dielectric profile of the structure of the periodic rectangular profile increased, the output profile of the half-sine $\left(T E_{10}\right)$ profile decreased, and the output amplitude increased. These results are strongly affected by the half-sine $\left(T E_{10}\right)$ input-wave profile and the locations of the rectangular profiles along $x$-axis and along $\mathrm{y}$-axis. The parameters are $\mathrm{a}=2 \mathrm{~cm}, \mathrm{~b}=2 \mathrm{~cm}, \mathrm{z}=15 \mathrm{~cm}, k_{0}=1671 / \mathrm{m}, \lambda=3.75 \mathrm{~cm}$, and $\beta=581 / m$.

The output fields for the periodic structure with four circular profiles along $\mathrm{x}$-axis and $\mathrm{y}$-axis (Figure 1(f)) are demonstrated in Figure 8(c and d) for $\epsilon_{r}=4$ and 10, respectively. The center of the first circle is located at the point $(0.25 \mathrm{a}, 0.25 \mathrm{~b})$, the center of the second circle is located at the point $(0.75 \mathrm{a}, 0.25 \mathrm{~b})$, the center of the third circle is located at the point $(0.25 \mathrm{a}, 0.75 \mathrm{~b})$, and the center of the firth circle is located at the point $(0.75 \mathrm{a}, 0.75 \mathrm{~b})$. The parameters are $\mathrm{a}=2 \mathrm{~cm}$, $\mathrm{b}=2 \mathrm{~cm}, \mathrm{z}=15 \mathrm{~cm}, k_{0}=1671 / \mathrm{m}, \lambda=3.75 \mathrm{~cm}$, and $\beta=581 / \mathrm{m}$.

These results are strongly affected by the half-sine $\left(T E_{10}\right)$ input-wave profile, the locations of the rectangular profiles (Figure 1(c)) or circular profiles (Figure 1(f)) along x-axis and along $\mathrm{y}$-axis, and the distance between the centers of the profiles. By increasing the parameter $\varepsilon_{r}$, the Gaussian shape of the output field increased.

\section{Conclusions}

The main objective of this research was to understand the influence of the periodic rectangular and circular profiles in the cross section along the straight rectangular waveguide on the output field. The second objective was to develop the technique to calculate two kinds of the proposed periodic profiles in the cross section. The calculations are based on using Laplace and Fourier transforms, and the output fields are computed by the inverse Laplace and Fourier transforms.

The contribution of the technique and the particular application is important to improve the method that is based on Laplace and Fourier transforms and their inverse transforms also for the discontinuous problems of the periodic rectangular and circular profiles in the cross section (and not only for the continuous profiles). The particular application is based on the $\omega_{\varepsilon}$ function. Thus, the proposed techniques are very effective in relation to the conventional methods because they allow the development of expressions in the cross section only according to the specific discontinuous problem.

All the graphical results were demonstrated as a response to a half-sine $\left(T E_{10}\right)$ input-wave profile and the periodic profiles in the cross section of the straight rectangular waveguide.

Three examples of periodic rectangular profiles are shown in Figure 1(a-c), and three examples of periodic circular profiles are shown in Figure 1(d-f) in the cross section of the straight rectangular waveguide. It is very interesting to compare between two different techniques of the two different kinds of the profiles (rectangular and circular) in the cross section of the rectangular straight waveguide. 
Figure 5(a-c) relates to Figure 1(a) and Figure 5(d-f) relates to Figure 1(d). The output fields (Figure $6(\mathbf{a}-\mathbf{f})$ ) are strongly affected by the input-wave profile ( $T E_{10}$ mode), the periodic structure with the two rectangular profiles or the two circular profiles along x-axis, and the distance between the two centers of the rectangular or the circular profiles.

It is interesting to see a similar behavior of the output results in the cases of periodic rectangular profiles (Figure 5(a-c)) that relate to Figure 1(a) and in the cases of circular profiles (Figure 5(d-f)) that relate to Figure 1(d), where $\epsilon_{r}=3$ and 10, respectively. The behavior is similar, but not for every $\epsilon_{r}$, and the amplitudes of the output fields are different.

Figure 6(a-e) relates to Figure 1(b) and Figure 7(a-e) relates to Figure 1(e). The output fields (Figure 6(a-e) and Figure 7(a-e)) are strongly affected by the input-wave profile ( $T E_{10}$ mode), the periodic structure with the two rectangular profiles (Figure 1(b)) or circular profiles (Figure 1(e)) along y-axis, and the distance between the two centers of the profiles.

By changing only the parameter $\epsilon_{r}$ from 3 to 10, the relative profile of the output field is changed from a half-sine ( $\left.T E_{10}\right)$ profile to a Gaussian shape profile, as shown in Figure 6(d) and Figure 7(d). The output fields in Figure 6(e) and in Figure 7(e) demonstrate the periodic structure with the two rectangular and circular profiles for $\epsilon_{r}=3,5,7$, and 10 .

It is interesting to see a similar behavior of the output results in the cases of periodic rectangular profiles (Figure 6(a-e)) that relate to Figure 1(b) and in the cases of periodic circular profiles (Figure 7(a-e)) that relate to Figure 1(e), for every value of $\epsilon_{r}$, respectively. According to these output results, we see the similar behavior for every value of $\epsilon_{r}$, but the amplitudes of the output fields are different.

The results of the periodic structures of the output field along $\mathrm{x}$-axis and y-axis are demonstrated in Figure 8(a-d). Figure 8(a and b) relates to Figure 1(c) and Figure 8(c and d) relates to Figure 1(f). By increasing the parameter $\epsilon_{r}$, the output dielectric profile of the structure of the periodic rectangular profile increased, the output profile of the half-sine $\left(T E_{10}\right)$ profile decreased, and the output amplitude increased. The results are strongly affected by the halfsine $\left(T E_{10}\right)$ input-wave profile and the locations of the rectangular or circular profiles. The results show in general similar behavior of the output field, but not the same results, and also the amplitudes of the output fields are different.

The application is useful for straight waveguides in the microwave and the millimeter wave regimes, with periodic rectangular and periodic circular profiles in the cross section of the straight waveguide.

\section{Author details}

\section{Zion Menachem}

Address all correspondence to: zionm@post.tau.ac.il

Department of Electrical Engineering, Sami Shamoon College of Engineering, Beer Sheva, Israel 


\section{References}

[1] Chiang KS. Review of numerical and approximate methods for the modal analysis of general optical dielectric waveguides. Optical and Quantum Electronics. 1993;26:S113-S134

[2] Zhao S. Full-vectorial matched interface and boundary (MIB) method for the modal analysis of dielectric waveguides. Journal of Lightwave Technology. 2008;26:2251-2259

[3] Jiao CQ. Selective suppression of electromagnetic modes in a rectangular waveguide by using distributed wall losses. Progress in Electromagnetics Research Letters. 2011;22:119-128

[4] Mineo M, Carlo AD, Paoloni C. Analytical design method for corrugated rectangular waveguide SWS THZ vacuum tubes. Journal of Electromagnetic Waves and Applications. 2010;24:2479-2494

[5] Smartt CJ, Benson TM, Kendall PC. Exact transcendental equation for scalar modes of rectangular dielectric waveguides. Optical and Quantum Electronics. 1994;26:641-644

[6] Chen TT. Wave propagation in an inhomogeneous transversely magnetized rectangular waveguide. Applied Scientific Research. 1960;8:141-148

[7] Yeap KH, Tham CY, Yassin G, Yeong KC. Attenuation in rectangular waveguides with finite conductivity walls. Radioengineering. 2011;20:472-478

[8] Tian JS, Tian LD. Rigorous analysis of electromagnetic wave propagation in cylindrical dielectric waveguide array. International Journal of Infrared and Millimeter Waves. 2003; 24:1885-1891

[9] Sharma J. Full-wave analysis of dielectric rectangular waveguides. Progress in Electromagnetics Research. 2010;13:121-131

[10] Sukhinin SV. Waveguide effect in a one-dimensional periodically penetrable structure. Journal of Applied Mechanics and Technical Physics. 1990;31:580-588

[11] Sumathy M, Vinoy KJ, Datta SK. Analysis of rectangular folded-waveguide millimeterwave slow-wave structures using conformal transformations. Journal of Infrared, Millimeter, and Terahertz Waves. 2009;30:294-301

[12] Rothwell EJ, Temme A, Crowgey B. Pulse reflection from a dielectric discontinuity in a rectangular waveguide. Progress in Electromagnetics Research. 2009;97:11-25

[13] Bulgakov AA, Kostylyova OV, Meriuts AV. Electrodynamic properties of a waveguide with layered-periodic walls. Radiophsics and Quantum Electronics. 2005;48:48-56

[14] Lu W, Lu YY. Waveguide mode solver based on Neumann-to-Dirichlet operators and boundary integral equations. Journal of Computational Physics. 2012;231:1360-1371

[15] Eyges L, Gianino P. Modes of dielectric waveguides of arbitrary cross sectional shape. Journal of the Optical Society of America. 1979;69:1226-1235 
[16] Soekmadji H, Liao SL, Vernon RJ. Experiment and simulation on $T E_{10}$ cut-off reflection phase in gentle rectangular downtapers. Progress in Electromagnetics Research Letters. 2009;12:79-85

[17] Abbas Z, Pollard RD, Kelsall W. A rectangular dielectric waveguide technique for determination of permittivity of materials at W-band. IEEE Transactions on Microwave Theory and Techniques. 1998;46:2011-2015

[18] Hewlett SJ, Ladouceur F. Fourier decomposition method applied to mapped infinite domains: scalar analysis of dielectric waveguides down to modal cutoff. Journal of Lightwave Technology. 1995;13:375-383

[19] Binzhao C, Fuyong X. Analysis for new types of waveguide with Fourier's expansiondifferential method. International Journal of Infrared and Millimeter Waves. 2008;29:240-248

[20] Hernandez-Lopez MA, Quintillan M. Propagation characteristics of modes in some rectangular waveguides using the finite-difference time-domain method. Journal of Electromagnetic Waves and Applications. 2000;14:1707-1722

[21] Vaish A, Parthasarathy H. Analysis of rectangular waveguide using finite element method. Progress in Electromagnetics Research C. 2008;2:117-125

[22] Baganas K. Inhomogeneous dielectric media: wave propagation and dielectric permittivity reconstruction in the case of a rectangular waveguide. Journal of Electromagnetic Waves and Applications. 2002;16:1371-1392

[23] Menachem Z, Jerby E. Transfer matrix function (TMF) for propagation in dielectric waveguides with arbitrary transverse profiles. IEEE Transactions on Microwave Theory and Techniques. 1998;46:975-982

[24] Menachem Z, Tapuchi S. Influence of the spot-size and cross-section on the output fields and power density along the straight hollow waveguide. Progress in Electromagnetics Research. 2013;48:151-173

[25] Vladimirov V. Equations of Mathematical Physics. New York (NY): Marcel Dekker, Inc., 1971 